\title{
A neural network-based electromyography motion classifier for upper limb activities
}

\author{
Karan Veer**, Tanu Sharma ${ }^{\dagger}$ and Ravinder Agarwal* \\ *Electrical and Instrumentation Engineering Department \\ Thapar University \\ ${ }^{\dagger}$ Computer Science Engineering Department \\ GCET, Ropar 174001, India \\ \$karan.una@gmail.com
}

Received 19 October 2015

Accepted 20 December 2015

Published 13 April 2016

\begin{abstract}
The objective of the work is to investigate the classification of different movements based on the surface electromyogram (SEMG) pattern recognition method. The testing was conducted for four arm movements using several experiments with artificial neural network classification scheme. Six time domain features were extracted and consequently classification was implemented using back propagation neural classifier (BPNC). Further, the realization of projected network was verified using cross validation (CV) process; hence ANOVA algorithm was carried out. Performance of the network is analyzed by considering mean square error (MSE) value. A comparison was performed between the extracted features and back propagation network results reported in the literature. The concurrent result indicates the significance of proposed network with classification accuracy (CA) of $100 \%$ recorded from two channels, while analysis of variance technique helps in investigating the effectiveness of classified signal for recognition tasks.
\end{abstract}

Keywords: ANN; SEMG; arm recognition; statistics; classification; RMS; upper arm activities.

\section{Introduction}

The technology of surface electromyogram (SEMG) recording is relatively new and has been used in various aspects of biomedical applications and can be derived from muscles using single or multiple channels. ${ }^{1}$ Since SEMG signal is deterministic and random in nature ${ }^{2}$ so its direct use is not recommended in prosthetic development. In the last two decades ${ }^{3-10}$ it has become successfully practical to classify these signals recorded from forearm muscles. However, the processing and recognition of signals recorded from upper arm muscles is an open research since last decade. The interesting issues in classifying upper limb signals are to investigate, what are the activities to be

\$Corresponding author.

This is an Open Access article published by World Scientific Publishing Company. It is distributed under the terms of the Creative Commons Attribution 4.0 (CC-BY) License. Further distribution of this work is permitted, provided the original work is properly cited. 
performed against multiple electrode sites; signal correlation within the group (WG) and between the group (BG) and finally what patterns could be segregated before applying classification.

With computers and software becoming more powerful tools to process complex algorithms on large data at high speed, SEMG features can be computed in numerical form from a finite length time interval which changes as a function of time, i.e., a voltage or a frequency. There are still limitations in the detection and characterization of existing nonlinearities in the SEMG signal, estimation of the phase, acquiring exact information due to derivation from normality. ${ }^{11-17}$ Further, SEMG signals reflect the nonlinear characteristics nature, so it becomes necessary to recognize motion commands before controlling the prosthetic and hence artificial neural network plays a vital role to this end as it makes the classification of SEMG signals possible. $^{18}$

Recent research has reported on the use of single electrode sensor channel surface EMG signals rather than using multichannel sensors. However, this entails the problem that when using EMG to identify complex actions it is necessary to map the EMG signals corresponding to the contractions of different muscles. ${ }^{19}$

Since the success of a myoelectric control scheme depends largely on the classification accuracy (CA) hence this concept has been used for the development of myoelectric prosthesis control systems obtained by classification of EMG signals. $\mathrm{Hu}$ and Nenov $^{20}$ compared the performance of two feature extraction methods for multichannel EMG based arm movement classification. Tsenov et al. ${ }^{21}$ also utilized two EMG electrodes to detect four finger movements using time domain features and neural networks classifiers achieving nearly $93 \%$ accuracy. Choi and $\mathrm{Kim}^{22}$ investigated to design an assistive real time system for the upper limb disabled. Wojtczak et $a ._{.}{ }^{23}$ used artificial neural networks to classify EMG signals to control multifunction prosthesis. Finger motions discrimination was emphasized most. Cipriani et $a ._{.}{ }^{24}$ reports real-time experiments on both able-bodied and amputees participants.

This issue is still a part of open research throughout the world for the researchers, since there is no well recognized outcome measure used throughout the world for the classification purpose. During study, four independent kinds of commonly used arm movements were selected. In addition, two arm muscle locations were selected for detecting the meaningful SEMG signals.

The whole process has been divided into four steps:

(i) Signal detection and it's presentation;

(ii) Feature extraction;

(iii) Recognition of patterns using neural network;

(iv) Cross validation (CV) using analysis of variance.

\section{Material and Methods}

Since many previous studies have presented multiple SEMG electrode configurations for achieving better (CA) of myoelectric signals, ${ }^{18}$ so, the objective of this work is to classify upper arm movements recorded from two SEMG channels using back propagation neural classifier (BPNC), and to evaluate the feasibility of recorded myoelectric signals using ANOVA technique.

The block diagram of the proposed study is shown in Fig. 1, which is composed of SEMG signal detection, feature extraction, neural network classification and effectiveness of classified data for recognition of arm gestures using ANOVA. The processing of data consists of three major stages: At

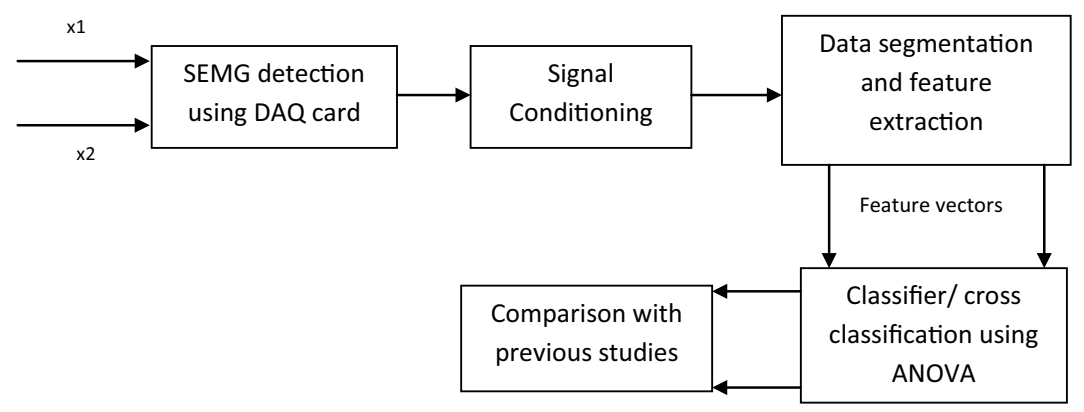

Fig. 1. Schematic of upper arm classification. 
first, arm motions are sensed using noninvasive electrodes, next in the second step; the activities of the individual muscles are decomposed and classified by back propagation neural network (BPNN) classifier. Finally, in last step, the particular arm activities are cross classified using repeated factorial analysis of variance technique.

\subsection{Subject's detail}

The detail of volunteers who participated in study; acquisition setup to measure the SEMG signals; sampling rate; signal filtering; signal processing procedure had been discussed in author's previous work..$^{1,13,17}$ An epoch of 3s from each muscle point was used while recording SEMG signals from upper arm residual muscles; namely biceps and triceps brachii, respectively. A total of four activities were performed on two muscle points independently. The participants were asked to perform one category of movement 3 times in each trail. A rest time of $5 \mathrm{~min}$ were allowed in between to minimize the potential effects of mental and muscle fatigue.

\section{Computation of Features}

For the prosthetic devices the controllers based on the available technologies are complex and bulky. Since various mathematical and artificial intelligence algorithms have made it practical to develop advanced analysis techniques, so six parameters were evaluated for carrying out the interpretation of SEMG signal task efficiently. The calculations parameters to be employed for classification task are shown in Table 1.

Table 1. Calculated SEMG parameters.

\begin{tabular}{|c|c|c|}
\hline Sr. No. & Parameters & Definitions \\
\hline 1 & $\begin{array}{l}\text { Root mean } \\
\text { square }\end{array}$ & Root mean square value of an epoch \\
\hline 2 & $\begin{array}{l}\text { Standard } \\
\text { deviation }\end{array}$ & $\begin{array}{l}\text { Variation or dispersion from the } \\
\text { average value }\end{array}$ \\
\hline 3 & Variance & Measure of the EMG signal's power \\
\hline 4 & $\begin{array}{l}\text { Simple square } \\
\text { integral }\end{array}$ & $\begin{array}{l}\text { Summation of the square values of } \\
\text { the amplitude signal samples }\end{array}$ \\
\hline 5 & $\begin{array}{l}\text { Integrated } \\
\text { EMG }\end{array}$ & $\begin{array}{l}\text { Summation of absolute values of the } \\
\text { EMG signal amplitude }\end{array}$ \\
\hline 6 & Power & $\begin{array}{l}\text { Relationship between total electrical } \\
\text { energy output of SEMG signal and } \\
\text { muscle contraction }\end{array}$ \\
\hline
\end{tabular}

\subsection{Pattern classification}

Artificial neural networks are built from single neurons grouped in layers. Neurons from one layer are connected to every neuron in another layer. The structure of the artificial neural network and neuron model is presented in Fig. 2. The neural classifier application was built based on a multithreaded structure in which three main parts can be distinguished: Data acquisition with the highest priority, the analysis and user interface with the lowest priority.

The architecture of neural network consisted of three layers: input, hidden and output as shown in Fig. 2.

The CA of designed Network actually depends on the feature set, network structure and training algorithm. Steps for improving network structure and overfitting, input data is divided as; $75 \%$ for training and $15 \%$ for validation and $10 \%$ for testing. The learning process for neuron model $(6: 3: 1)$ for classification is to be carried out as follows:

- All participated subject attempts to make four independent upper arm movement;

- Since there is no general rule for computing number of neurons in hidden layer, so this numbers should be small to simplify the computation and to reduce the risk of overfitting;

- Patterns received from controlled signals are fed to neural network;

- Network reiterates the procedure in exercising SEMG patterns from arm movement for increased network performance with minimum MSE;

- Trained network is evaluated for classifying upper arm movements.

\subsection{One way analysis of variance statistical technique}

Since parameter "RMS" provides the higher performance in detection of arm movements, so has

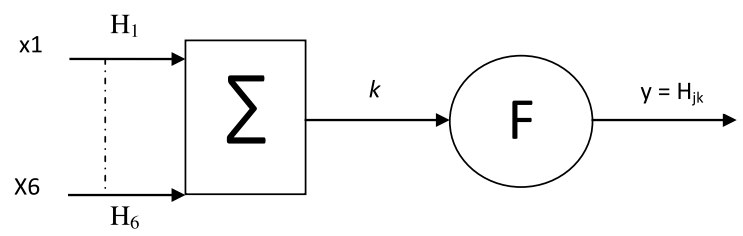

Fig. 2. Block diagram for Artificial neuron model (6:3:1) for classification. 


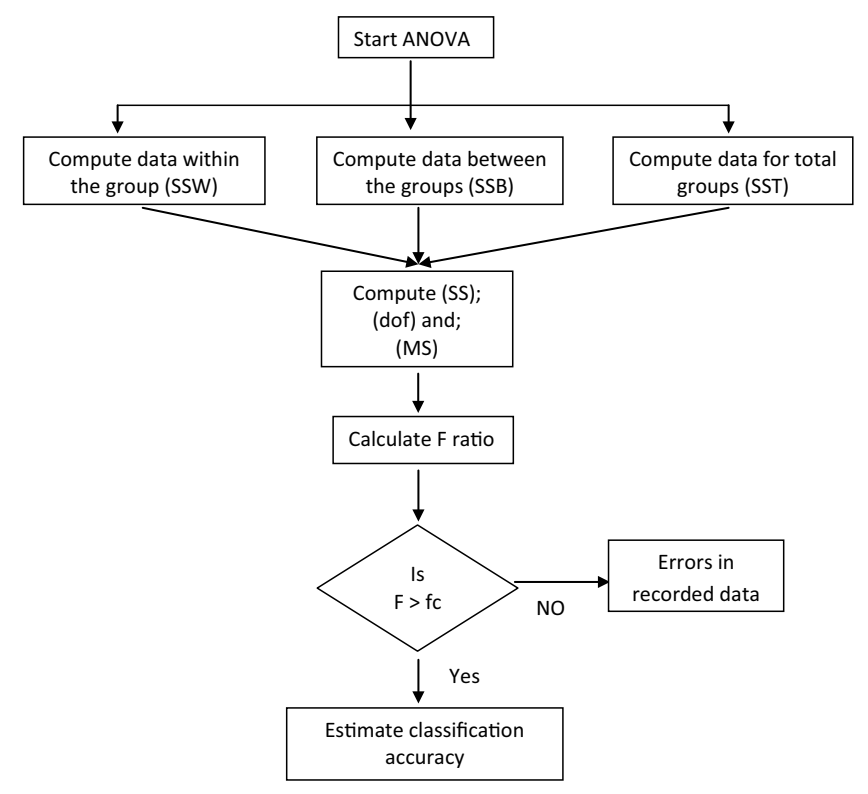

Fig. 3. Block diagram for analysis of variance steps.

been applied to interpret the effectiveness of recorded signal. The block diagram of ANOVA model is presented in Fig. 3. The major points in the evaluation of technique consist of following issues:

(1) Four independent Groups (G1 - G4);
(2) Seven amputed volunteers for each group;

(3) Two upper arm muscle locations, biceps and triceps brachii;

\section{Result}

As SEMG is time and force dependent signals, so signal's interpretation becomes necessary before defining the characteristic properties. The SEMG signals were segmented by the length of 3000 samples and also the amplitudes of segmented signals were normalized for avoiding the effect of different amplitude scale. The block diagram for the entire system including bipolar electrode configuration, amplifiers, DAQ are as depicted in Fig. 4.

A simulated Labview soft scope aided code was designed and then executed for conducting SEMG signal force relationship. Tables 2 and 3 tabulated the average comparison of extracted parameters being analyzed from biceps and triceps muscles for participated volunteers.

These time domain features has been used in converting recorded data into amplitude envelope, making it easier to view and consequently to make concerned decision. Figures 5 and 6 helps in

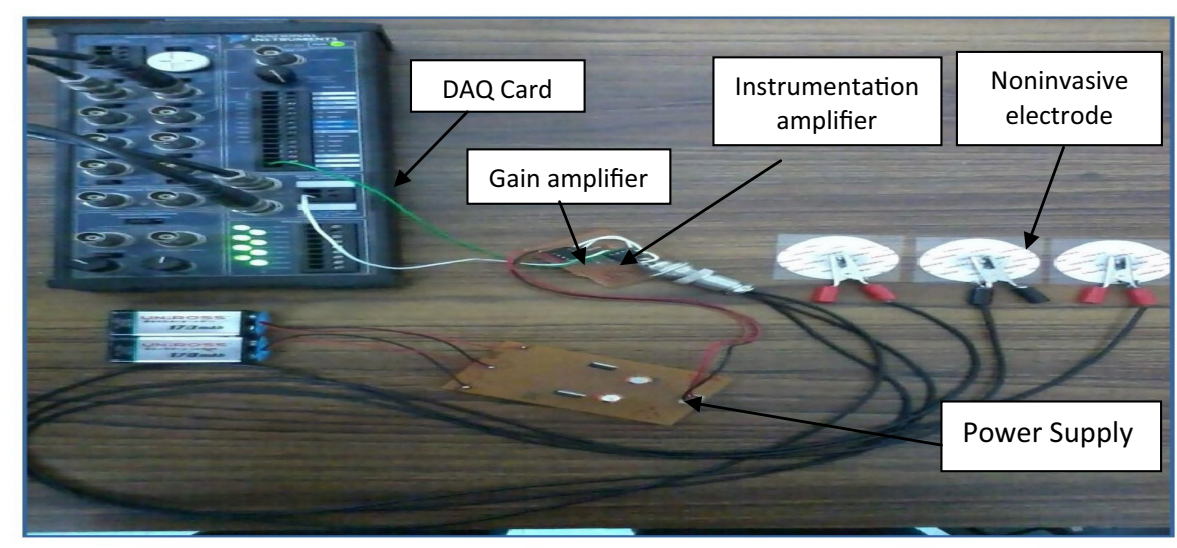

Fig. 4. Figure of the entire testing system.

Table 2. Feature extracted values for biceps muscle.

\begin{tabular}{lcccccc}
\hline \multicolumn{7}{c}{ Biceps muscle } \\
\hline Activities & RMS & VAR & SSI (/1000) & SD & P & IEMG (/1000) \\
\hline elbow extension (ee) & 0.15 & 0.02 & 31 & 0.10 & 5 & 191 \\
elbow flexion (ef) & 0.57 & 0.22 & 558 & 0.45 & 86 & 1016 \\
abduction (abd) & 0.18 & 0.03 & 49 & 0.12 & 6 & 277 \\
adduction (add) & 0.15 & 0.02 & 29 & 0.10 & 5 & 215 \\
\hline
\end{tabular}


Table 3. Feature extracted values for triceps muscle.

\begin{tabular}{lcccccc}
\hline \multicolumn{7}{c}{ Triceps muscle } \\
\hline Activities & RMS & VAR & SSI & SD & P & IEMG \\
\hline elbow extension (ee) & 0.16 & 0.02 & 27 & 0.10 & 8 & 210 \\
elbow flexion (ef) & 0.22 & 0.04 & 81 & 0.15 & 18 & 340 \\
abduction (abd) & 0.24 & 0.05 & 124 & 0.18 & 17 & 444 \\
adduction (add) & 0.41 & 0.10 & 268 & 0.29 & 43 & 661 \\
\hline
\end{tabular}

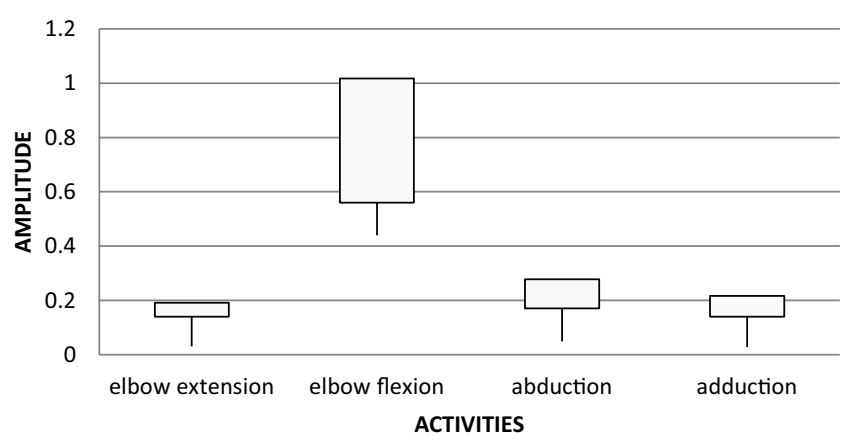

Fig. 5. Comparison of $\mathrm{V}_{\text {rms }}$ values for bicep movements.

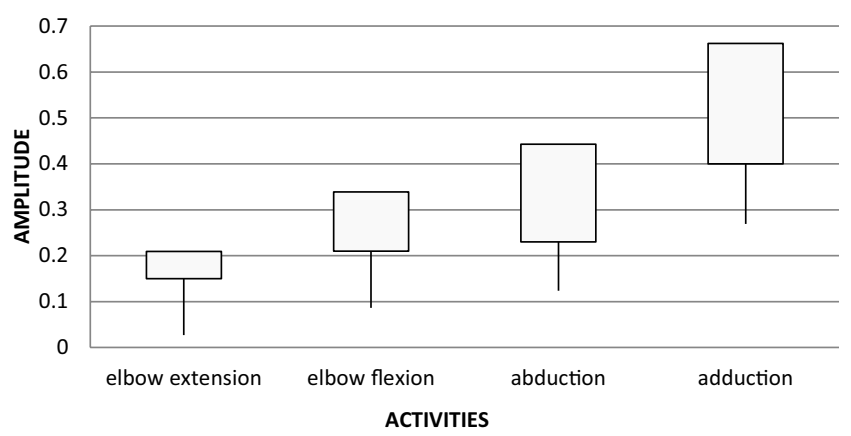

Fig. 6. Comparison of $\mathrm{V}_{\mathrm{rms}}$ values for tricep movements. evaluating the effectiveness of recorded data for biceps/triceps movements, respectively and would play a vital role in segregating recorded SEMG data prior to conduct ANN and ANOVA classification.

\subsection{Results using ANN classifier}

Back propagation supervised learning algorithm has been used for classifying motion patterns available from different four types of activities since it helps in minimizing MSE with improved performance. Further, subjects were trained thoroughly before performing four arm activities for obtaining efficient training sets. Six statistical time and time-frequency based features (Tables 2 and 3) from SEMG signals are fed as inputs to neural network.

The statistical analyses in terms of mean square error (MSE) were investigated for verifying the response and performance of the network. The network performance is best with lowest MSE, and is 0.04242 and 0.0000001 for train and then retraining stage. During experiment it was found that response time and complexity of the network was optimum for 3 neurons in the hidden layer. The training, validation, testing and overall classification performance of the proposed network is depicted in Fig. 7. From confusion matrix, the results occurring in diagonal form are the correct classification rates while the results outside the diagonal are the errors. The average of MSE value with standard deviation error for the four upper arms is shown in Fig. 8. If one considers the class 2, '21' numbers of upper arm movements are correctly classified which means the average classification for this class is $100 \%$ against total subjects and is

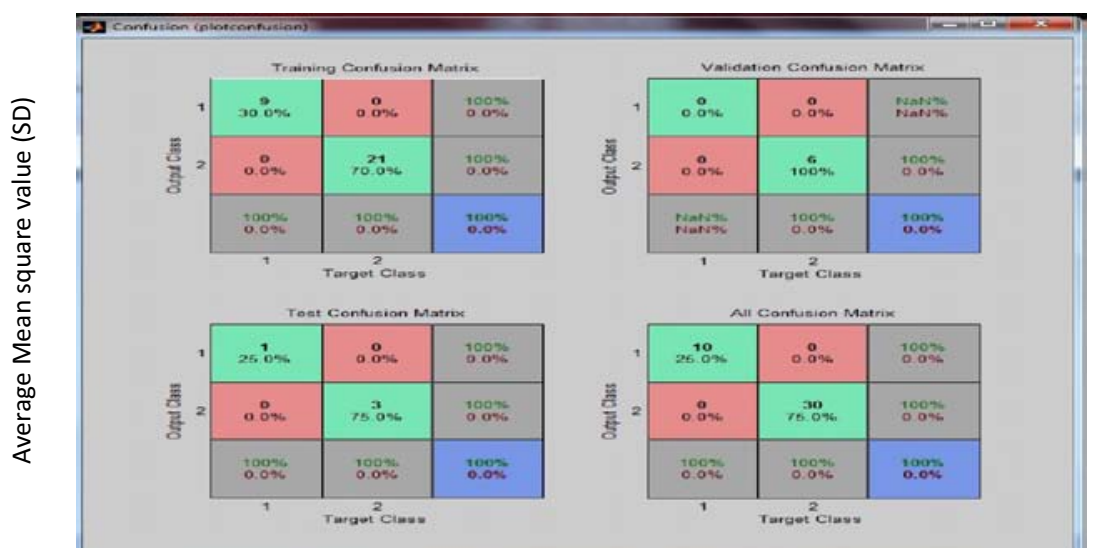

Fig. 7. Neural network based confusion matrix. 


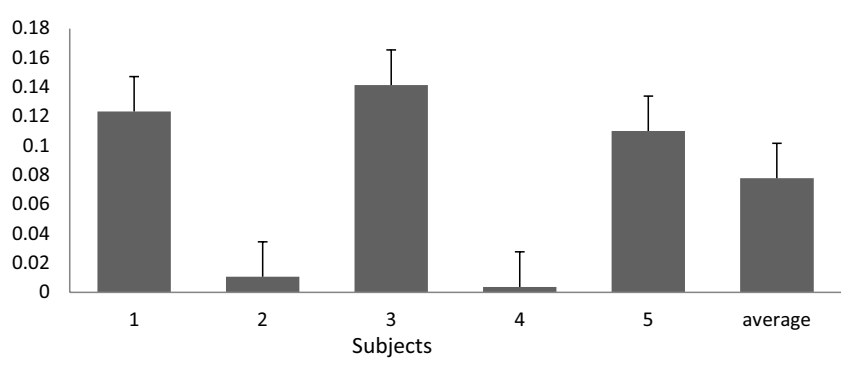

Fig. 8. Average mean square value (\%) with SD for the four upper arm movements.

Table 4. List of individual classification accuracies with MSE for participated subjects.

\begin{tabular}{ccc}
\hline SUBJECTS & CA (100\%) & MSE \\
\hline 1 & 87.5 & $1.23 \mathrm{E}-01$ \\
2 & 100 & $1.07 \mathrm{E}-07$ \\
3 & 100 & $1.42 \mathrm{E}-01$ \\
4 & 100 & $3.80 \mathrm{E}-08$ \\
5 & 100 & $1.10 \mathrm{E}-07$ \\
Average & 97.50 & $5.30 \mathrm{E}-02$ \\
\hline
\end{tabular}

$97.50 \%$ from average of participated subjects (Table 4), respectively.

\subsection{Results using statistical technique}

Since CV is often used for comparing two or more learning models to estimate which model performs best for the classification purposes, hence repeated factorial analysis of variance algorithm has been implemented.
The basic principle is to test for differences among the means of the populations by examining the amount of variation within each of these samples, relative to the amount of variations between the samples. Eventually one has to make two estimates of population variance, i.e., one based on between samples variance and other is based on within samples variance. Finally, two aforesaid estimates of population variances are compared against $\mathrm{F}$-test as follows:

$\mathrm{F}=$ Estimate of variance between samples/ Estimate of variance within samples.

The standard one-way ANOVA hypothesis tests are valid under the following assumptions:

(1) The treatment populations must be normal,

(2) The treatment populations must all have the same variance.

A total of 15 volunteers participated in this part of study. The overall statistical results for the different activities on biceps brachii and triceps brachii muscles have been tabulated in Tables 5 and 6 . From the tables, it was concluded that there was significant difference in amplitude gain across different motions as $\mathrm{F}(3,24)=25.78, p<0.05$ and $\mathrm{F}$ $(3,24)=9.33, p<0.05$ for two independent muscles against critical value (3.01). Since F ratio is greater than critical value (fc), so it was observed that there exists significant difference between the groups (SSB) than within groups (SSW). The statistical results confirm the dependency of multiple arm movements on defined locations in terms of amplitude estimation.

Table 5. ANOVA statistics for four different arm movements on biceps muscle.

\begin{tabular}{lcccccc}
\hline Group No. & Criterion & Mean & $(x \text {-mean })^{2}$ & $P$ & F ratio & Critical value \\
\hline 1 & Fisher algorithm & 0.62 & 0.01 & 0.0000011 & $\mathbf{2 5 . 7 8}$ & 3.01 \\
2 & Fisher algorithm & 2.78 & 0.07352 & 0.0000011 & & \\
3 & Fisher algorithm & 0.83 & 0.00472 & 0.0000011 & & \\
4 & Fisher algorithm & 0.69 & 0.00868 & 0.0000011 & & \\
\hline
\end{tabular}

Table 6. ANOVA statistics for four different arm movements on triceps muscle.

\begin{tabular}{lcccccc}
\hline Group No. & Criterion & Mean & $(x \text {-mean })^{2}$ & $P$ & F ratio & Critical value \\
\hline 1 & Fisher algorithm & 0.70 & 0.0252 & 0.00028 & $\mathbf{9 . 3 3}$ & 3.01 \\
2 & Fisher algorithm & 1.03 & 0.0665 & 0.00028 & & \\
3 & Fisher algorithm & 1.33 & 0.0385 & 0.00028 & & \\
4 & Fisher algorithm & 2.01 & 0.0603 & 0.00028 & & \\
\hline
\end{tabular}


Table 7. Summary of neural network based signal classification from EMG.

\begin{tabular}{llll}
\hline Sr. No. & \multicolumn{1}{c}{ Method } & Number of channels & \multicolumn{1}{c}{ Classification accuracy } \\
\hline 1 & NMF neural classifier & & \multicolumn{1}{c}{${ }^{18}$} \\
2 & Levenberg - Marquardt neural network $^{26}$ & 2 & $95 \%$ for simple and $87 \%$ for complex flexions \\
3 & SMC and SCC classifier & 1 & $88.4 \%$ \\
4 & Bayesian data fusion approach & 28 & $84 \%$ and $81 \%$ \\
5 & This present work, BPNN & 2 & $90 \%$ \\
\hline
\end{tabular}

\section{Previous Work}

The main issues to be noted while comparing the results against previous studies are electrode placement sites, acquisition set ups; subject protocols and the ways of presenting recoded data; and there is always nonuniformity in these parameters hence it is hard to compare results. However, trending information could be helpful in analyzing the effectiveness of one's recorded data. ${ }^{25}$ The results of previous studies have been compared (Table 7).

\section{Discussion}

Since main interest is to investigate the role of muscle fiber during voluntary contractions, the estimation of variation in the amplitude of SEMG needs to be quantified for interpretation of signals, so analytical features were extracted and evaluated before classifying arm movements using ANN classifier. Dual channel SEMG work class was carried out and experiments being conducted to four different upper arm movements collected from total subjects proved the feasibility of the proposed network with $100 \%$ of CA using back propagation neural network. The proposed neural network also helped in reducing the time required for classification. Since, each recorded data set has one single pattern, so it becomes essential to separate and classify these patterns. Hence proposed algorithm classified four movements significantly with $100 \%$ accuracy and 0.04242 mean square value. Next, one way repeated factorial analysis of variance technique helps in segregating different operations that could be easily realized by prosthetic devices.

\section{Conclusion}

From Figs. (5) and (6), one can easily conclude that elbow flexion and adduction movements against biceps and triceps muscle location are the best one and can be used for designing prosthetic device. Further neural network with back propagation algorithm was trained with extracted features to classify arm movements. The result suggests that employing first and second trail; the optimized neural network can significantly classify the SEMG signals with average classification rate of $100 \%$. After comparing with existing methods, the proposed method exhibited higher $\mathrm{CA}$ and more robustness. Finally, it is concluded that neural network algorithm makes recognition more effective and efficient for classification purposes while statistical technique of ANOVA, additionally is a significant approach to report the effectiveness of recorded data. In future studies, more powerful algorithms will be applied for the interpretation of upper limb signals.

\section{References}

1. V. Karan, "Interpretation of surface electromyograms to characterize arm movement," Instrum. Sci. Technol. 42, 513-521 (2014).

2. J. Zahak, W. Asim, N. Shaheryar, K. Shahryar, I. Javaid, M. Adnan, S. Umar, Motor drive using surface electromyography for flexion and extension of finger and hand muscles, 4th Int. Conf. Biomedical Engineering and Informatics, Shanghai, pp. 1287-1291 (2011).

3. Y. Huang, K. B. Englehart, B. Hudgins, A. C. Chan, "A Gaussian mixture model based classification scheme for myoelectric control of powered upper limb prosthesis," IEEE Trans. Biomed. Eng. 52, 18011811 (2005).

4. R. N. Khushaba, A. Al-Ani, A. Al-Jumaily, "Orthogonal fuzzy neighborhood discriminant analysis for multifunction myoelectric hand control," IEEE Trans. Biomed. Eng. 57, 1410-1419 (2010).

5. J. Demsar, "Statistical comparisons of classifiers over multiple data sets," J Mach. Learn. Res. 7, 1-30 (2006).

6. R. G. Naik, D. Kumar, "Subtle electromyographic pattern recognition for finger movements: A pilot 
study using BSS techniques," J. Mech. Med. Biol. 12, 1-19 (2012).

7. L. Hargrove, K. Englehart, B. Hudgins, "A training strategy to reduce classification degradation due to electrode displacements in pattern recognition based myoelectric control," Biomed. Signal Process Control 3, 175-180 (2008).

8. T. Sharma, K. Veer, R. Agarwal, "Non invasive technique based evaluation of electromyogram signals using statistical algorithm," Int. J. Adv. Res. Comput. Eng. Technol. 3, 1987-1991 (2014).

9. R. G. Naik, D. Kumar, M. Palaniswami, "Signal processing evaluation of myoelectric sensor placement in low-level gestures: Sensitivity analysis using independent component analysis," Expert Syst. 31, 91-99 (2014).

10. K. Veer, "Experimental study and characterization of semg signals for upper limbs," Fluct. Noise Lett. 14, 150028 (2015).

11. P. Geethanjali, K. Ray, "Statistical pattern recognition technique for improved real-time myoelectric signal classification," Biomed. Eng., Appl. Basis Comm. 25, 1-9 (2013).

12. P. Kaufmann, K. Englehart, M. Platzner, Fluctuating EMG signals: Investigating long-term effects of pattern matching algorithms, Proc. Annu. Int. Conf. IEEE Eng. Med. Biol. Soc., Buenos Aires, Argentina, pp. 6357-6360 (2010).

13. K. Veer, R. Agarwal, "Simulated processing of electromyogram signal for design of prosthetic devices using statistical technique," J. Appl. Stat. 42, 1591-1601 (2015).

14. D. A. Gabriel, G. Kamen, "Experimental and modeling investigation of spectral compression of biceps brachii SEMG activity with increasing force levels," J. Electromyogr. Kinesiol. 19, 437-448 (2009).

15. K. Holzbaur, W. Murray, G. Gold, S. Delp, "Upper limb muscle volumes in adult subjects," J. Biomech. 40, 742-74 (2007).

16. A. Phinyomark, P. Phukpattaranont, C. Limsakul, "Feature extraction and reduction of wavelet transform coefficients for EMG pattern classification," Electron. Electr. Eng. Signal Technol. 6, 27-32 (2012).

17. K. Veer, "A technique for classification and decomposition of muscle signal for control of myoelectric prostheses based on wavelet statistical classifier," Measurement 60, 283-291 (2015).
18. R. Ahsan Md., I. M. Ibrahimy, O. O. Khalif, "Electromygraphy (EMG) signal based hand gesture recognition using artificial neural network (ANN)," 4th Int. Conf. Mechatronics (ICOM), Kuala Lumpur pp. 1-6 (2011).

19. A. Phinyomark, P. Phukpattaranont, C. Limsakul, "Fractal analysis features for weak and singlechannel upper-limb EMG signals," Expert Syst. Appl. 39, 11156-11163 (2012).

20. X. Hu, V. Nenov, "Multivariate AR modeling of electromyography for the classification of upper arm movements," Clin. Neurophysiol. 115, 1276-1287 (2004).

21. G. Tsenov, A. H. Zeghbib, F. Palis, N. Shoylev, V. Mladenov, Neural networks for online classification of hand and finger movements using surface EMG signals, Proc. 8th Seminar on Neural Network Applications in Electrical Engineering, University of Belgrade, Serbia, pp. 167-171 (2006).

22. C. Choi, J. Kim, A real-time EMG-based assistive computer interface for the upper limb disabled, Proc. 2007 IEEE 10th Int. Conf. Rehabilitation Robotics, Noordwijk (2007).

23. P. Wojtczak, T. G. Amaral, O. P. Dias, A. Wolczowski, M. Kurzynski, "Hand movement recognition based on biosignal analysis," Eng. Appl. Artificial Intell. 22, 608-615 (2009).

24. C. Cipriani, C. Antfolk, M. Controzzi, G. Lundborg, B. Rosen, M. C. Carrozza, "Online myoelectric control of a dexterous hand prosthesis by transradial amputees," IEEE Trans. Neural Syst. Rehabil. Eng. 19, 260-270 (2011).

25. M. Hariharan, Y. C. Fook, R. Sindhu, I. Bukhari, Y. Sazali, "A comparative study of wavelet families for classification of wrist motions," Comp. Electr. 38, 1798-1807 (2012).

26. M. I. Ibrahimy, Md. R. Ahsan, O. O. Khalifa, "Design and optimization of levenberg-marquardt based neural network classifier for EMG signals to identify hand motions," Meas. Sci. Rev. 13, 142151 (2013).

27. H. Parsaei, J. F. Nezhad, W. D. Stashuk, H. A. Wright, "Validating motor unit firing patters extracted by EMG signal decomposition," Med. Biol Eng Comput. 49, 649-658 (2011).

28. N. R. Ganesh, N. T. Hung, "Nonnegative matrix factorization for the identification of EMG finger movements: evaluation using matrix analysis," IEEE J. Biomed. Health Inform. 19, 478-485 (2015). 